\title{
Exact Finite-Difference Schemes for $d$-Dimensional Linear Stochastic Systems with Constant Coefficients
}

\author{
Peng Jiang, ${ }^{1}$ Xiaofeng Ju, ${ }^{2}$ Dan Liu, ${ }^{3}$ and Shaoqun Fan ${ }^{4}$ \\ ${ }^{1}$ Harbin Institute of Technology, Room 405, Apartment A11, 92 Xi Dazhi Street, Harbin, Heilongjiang 150001, China \\ ${ }^{2}$ School of Management, Harbin Institute of Technology, Room 225, 92 Xi Dazhi Street, Harbin, Heilongjiang 150001, China \\ ${ }^{3}$ Qingdao Technical College, 369 Qiantangjiang Road, Qingdao Economic and Technological Development Zone, \\ Qingdao, Shandong 266555, China \\ ${ }^{4}$ China Telecom Beijing Research Institute, 708 Guanhua Building, No. 118 Xizhimennei Street, Xicheng District, Beijing 100035, China
}

Correspondence should be addressed to Peng Jiang; peng.jiang.hit@gmail.com

Received 1 September 2013; Accepted 28 October 2013

Academic Editor: Jesus Vigo-Aguiar

Copyright (C) 2013 Peng Jiang et al. This is an open access article distributed under the Creative Commons Attribution License, which permits unrestricted use, distribution, and reproduction in any medium, provided the original work is properly cited.

The authors attempt to construct the exact finite-difference schemes for linear stochastic differential equations with constant coefficients. The explicit solutions to Itô and Stratonovich linear stochastic differential equations with constant coefficients are adopted with the view of providing exact finite-difference schemes to solve them. In particular, the authors utilize the exact finitedifference schemes of Stratonovich type linear stochastic differential equations to solve the Kubo oscillator that is widely used in physics. Further, the authors prove that the exact finite-difference schemes can preserve the symplectic structure and first integral of the Kubo oscillator. The authors also use numerical examples to prove the validity of the numerical methods proposed in this paper.

\section{Introduction}

Certainty barely exists as a natural phenomenon in isolation. On the contrary, the phenomenon of certainty is an integral part of a complex environment full of interrelations and interactions. Physics is the general analysis of nature, including elements of matter, motion, space, and time, all of which are relevant to the concept of certainty and uncertainty. In order to capture a clear understanding of physical movements in the natural world by using modeling techniques, it is inevitable to take the effects, as the result of the uncertainty upon any development course of any entities, into full consideration. The stochastic differential equations are one of the best known techniques to depict physical movement in precision. For instance, statistical physics is based on the ergodicity assumption of the development of a system and stochastic differential equations can give perfect solutions to such scenarios. Moreover, the stochastic differential equations and relevant applications have been applied in such a variety of scientific fields such as stochastic control, stochastic neural network, financial economic studies, species dynamics, and electronic engineering [1-5]. In this regard, it is of crucial importance to explore stochastic differential equations. Major research findings of the stochastic differential equations can be found in $[4,5]$.

Although Mao [4] and Kloeden and Platen [6] have managed to calculate the expression of the explicit solution to certain types of stochastic differential equations, it is not always possible to derive the explicit solution to equations of such kind. Strong nonlinearity and coupling characteristics of stochastic differential equations are the major reason and, in this sense, it is of significant importance to establish an effective numerical method [6-15] that is able to make rational judgment by using computer simulations. In saying so, a new branch of computational mathematics is emerging as the numerical solutions to stochastic differential equations. The fundamental theories in this area can be found in $[6,15]$.

In recent years, the exact finite-difference schemes of ordinary differential equations have captured more and more attention from the academia. Such exact finite-difference schemes are the same to the solutions to the original equations, whilst being able to retain the characteristics of 
the solutions. Further, such exact finite-difference schemes can be utilized to rationalize the construction of nonstandardized finite-difference schemes [16], for example, the exponentially fitted method [17-19]. As is well known, the linear ordinary differential equations with constant coefficients involve an exact finite-difference scheme (see $[16,20$, 21]) and some scholars have discussed exact discretizations of other systems, for example, Vigo-Aguiar and Ferràndiz [22], Cieśliński [23, 24], Sakamoto et al. [25], Mickens and Washington [26], Roeger et al. [27-29], and so on. However, there are only a few published papers [30-32] discussing whether an exact finite-difference scheme exists for widely applied linear stochastic differential equations with constant coefficients. In order to bridge the gap, this paper aims to calculate the exact finite-difference schemes of general $d$-dimensional linear stochastic differential equations with constant coefficients and hence apply the results to the Kubo oscillator so as to prove the structure-preserving property of the exact finite-difference schemes, which is a stochastic sample from [24].

The following part of the paper is organized as follows. In Section 2, the authors will discuss the exact finite-difference schemes for Itô and Stratonovich type stochastic differential equations. In Section 3, the authors demonstrate how to apply the findings generalized from Section 2 to the Kubo oscillator. Finally, the authors will use numerical examples to illustrate the validity of the findings.

\section{The Exact Finite-Difference Schemes for Linear Stochastic Differential Equations with Constant Coefficients}

Let $(\Omega, F, P)$ be a complete probability space with a filtration $\left\{F_{t}\right\}$. The filtration $\left\{F_{t}\right\}$ is increasing and right continuous, and $F_{0}$ contains all $P$-null sets. Let $B(t)=\left(B_{1}(t)\right.$, $\left.B_{2}(t), \ldots, B_{m}(t)\right)^{T}$ be a standard $m$-dimensional Brownian motion defined on $(\Omega, F, P)$, whose increment $\Delta B_{k}=B_{k}(t+$ $h)-B_{k}(t)(k=1,2, \ldots, m)$ is a Gaussian random variable $N(0, h)$.

Then we discuss numerical methods for strong solutions to stochastic differential equations:

$$
\begin{gathered}
d x(t)=f(x(t)) d t \\
+\sum_{k=1}^{m} g_{k}(x(t)) d B_{k}(t), \quad t \geq 0, \\
\quad x(0)=x_{0} \in R^{d},
\end{gathered}
$$

where the deterministic term $f(x)$ is the drift coefficient and the stochastic terms $g_{k}(x)(k=1,2, \ldots, m)$ are diffusion coefficients. The solution of (1) can be written as

$$
\begin{aligned}
x(t)= & x_{0}+\int_{0}^{t} f(x(s)) d s \\
& +\sum_{k=1}^{m} \int_{t_{0}}^{t} g_{k}(x(s)) d B_{k}(s) .
\end{aligned}
$$

Given an equidistant discretization of the interval $[0, t]$ with grid points $t_{n}(n=1,2, \ldots, N)$ and letting $\xi_{n}=\theta t_{n}+$ $(1-\theta) t_{n-1}(\theta \in[0,1])$, the stochastic integrals in (2) can be calculated by the limit of the approximating sums in the mean square sense, as $N \rightarrow \infty$,

$$
\sum_{n=1}^{N} g_{k}\left(x\left(\xi_{n}\right)\right)\left(B_{k}\left(t_{n}\right)-B_{k}\left(t_{n-1}\right)\right) \quad(k=1,2, \ldots, m)
$$

The values of stochastic integrals depend on the choice of $\theta$ and the Itô integrals when $\theta=0$. The corresponding Itô type stochastic differential equations are the equations using the usual notation (1) and the Stratonovich integrals when $\theta=1 / 2$. The corresponding Stratonovich type stochastic differential equations are denoted by

$$
\begin{gathered}
d x(t)=f_{1}(x(t)) d t \\
+\sum_{k=1}^{m} g_{k}(x(t)) \circ d B_{k}(t), \quad t \geq 0, \\
x(0)=x_{0} \in R^{d} .
\end{gathered}
$$

The relationships between these two types of stochastic differential equations are given by

$$
\begin{aligned}
f_{1}(x(t))= & f(x(t)) \\
& -\frac{1}{2} \sum_{k=1}^{m} g_{k}^{\prime}(x(t)) g_{k}(x(t)) .
\end{aligned}
$$

The exact finite-difference schemes of $d$-dimensional linear stochastic differential equations with constant coefficients of Itô type can be discussed as

$$
\begin{gathered}
d x(t)=F x(t) d t+\sum_{k=1}^{m} G_{k} x(t) d B_{k}(t), \quad t \geq 0, \\
x(0)=x_{0} \in R^{d},
\end{gathered}
$$

where $F$ and $G_{k}(k=1,2, \ldots, m)$ are $d \times d$ matrices and $B_{k}(t)$ $(k=1,2, \ldots, m)$ are independent one-dimensional Brownian motion. Suppose that the matrices $F, G_{1}, G_{2}, \ldots, G_{m}$ are commutative; that is,

$$
F G_{k}=G_{k} F, \quad G_{k} G_{j}=G_{j} G_{k}, \quad k, j=1,2, \ldots, m .
$$

Let $h$ be the time step and $t_{n}=n h . \Delta B_{n}^{k}=B_{k}\left(t_{n+1}\right)-$ $B_{k}\left(t_{n}\right)(k=1,2, \ldots, m)$ denotes the increment of Brownian motion. Then the following theorem can be derived.

Theorem 1. Equation (6) admits the exact finite-difference schemes given by

$$
x_{n+1}=\exp \left\{\left(F-\frac{1}{2} \sum_{k=1}^{m} G_{k}^{2}\right) h+\sum_{k=1}^{m} G_{k} \Delta B_{n}^{k}\right\} x_{n},
$$

where $x_{n}$ is the exact value of solution $x(t)$ to (6) at $t=t_{n}$. 
Proof. Using the theories in [4, Chapter 3], the general solution of (6) can be expressed in terms of $F, G_{1}, G_{2}, \ldots, G_{m}$ as follows:

$$
x(t)=\exp \left\{\left(F-\frac{1}{2} \sum_{k=1}^{m} G_{k}^{2}\right) t+\sum_{k=1}^{m} G_{k} B_{k}(t)\right\} x_{0} .
$$

Inserting $t_{n}$ and $t_{n+1}$ into (9), it can be deduced that

$$
\begin{gathered}
x_{n}=x\left(t_{n}\right)=\exp \left\{\left(F-\frac{1}{2} \sum_{k=1}^{m} G_{k}^{2}\right) t_{n}+\sum_{k=1}^{m} G_{k} B_{k}\left(t_{n}\right)\right\} x_{0}, \\
x_{n+1}=x\left(t_{n+1}\right) \\
=\exp \left\{\left(F-\frac{1}{2} \sum_{k=1}^{m} G_{k}^{2}\right) t_{n+1}+\sum_{k=1}^{m} G_{k} B_{k}\left(t_{n+1}\right)\right\} x_{0} \\
=\exp \left\{\left(F-\frac{1}{2} \sum_{k=1}^{m} G_{k}^{2}\right)\left(h+t_{n}\right)\right. \\
\left.\quad+\sum_{k=1}^{m} G_{k}\left(\Delta B_{n}^{k}+B_{k}\left(t_{n}\right)\right)\right\} x_{0} \\
=\exp \left\{\left(F-\frac{1}{2} \sum_{k=1}^{m} G_{k}^{2}\right) h+\sum_{k=1}^{m} G_{k} \Delta B_{n}^{k}\right\} x_{n},
\end{gathered}
$$

which ends the proof.

Consider a $d$-dimensional linear stochastic differential equation with constant coefficient of Stratonovich type driven by $m$ independent one-dimensional Brownian motion:

$$
\begin{gathered}
d y(t)=A y(t) d t+\sum_{k=1}^{m} C_{k} y(t) \circ d B_{k}(t), \quad t \geq 0, \\
y(0)=y_{0} \in R^{d},
\end{gathered}
$$

where $A$ and $C_{k}(k=1,2, \ldots, m)$ are $d \times d$ matrices. It is assumed that the matrices $A, C_{1}, C_{2}, \ldots, C_{m}$ are commutative; that is,

$$
A C_{k}=C_{k} A, \quad C_{k} C_{j}=C_{j} C_{k}, \quad k, j=1,2, \ldots, m .
$$

The exact finite-difference schemes can be constructed by using the general solution of (11).

Theorem 2. Equation (11) admits the exact finite-difference schemes given by

$$
y_{n+1}=\exp \left\{A h+\sum_{k=1}^{m} C_{k} \Delta B_{n}^{k}\right\} y_{n},
$$

where $y_{n}$ is the exact value of solution $y(t)$ to (11) at $t=t_{n}$.

Proof. Firstly, we prove that the general solution to (11) is given by

$$
y(t)=\exp \left\{A t+\sum_{k=1}^{m} C_{k} B_{k}(t)\right\} y_{0} .
$$

Equation (14) follows (9) by a known relation (5) between Itô and Stratonovich type stochastic differential equations. Taking into account that $y_{n}=y\left(t_{n}\right)$ and, in particular, $y(0)=$ $y_{0}$, it can be obtained that

$$
\begin{aligned}
y_{n} & =y\left(t_{n}\right)=\exp \left\{A t_{n}+\sum_{k=1}^{m} C_{k} B_{k}\left(t_{n}\right)\right\} y_{0} \\
y_{n+1} & =y\left(t_{n+1}\right) \\
& =\exp \left\{A t_{n+1}+\sum_{k=1}^{m} C_{k} B_{k}\left(t_{n+1}\right)\right\} y_{0} \\
& =\exp \left\{A\left(h+t_{n}\right)+\sum_{k=1}^{m} C_{k}\left(\Delta B_{n}^{k}+B_{k}\left(t_{n}\right)\right)\right\} y_{0} \\
& =\exp \left\{A h+\sum_{k=1}^{m} C_{k} \Delta B_{n}^{k}\right\} y_{n} .
\end{aligned}
$$

This completes the proof of the theorem.

Remark 3. Although the existence of the exact solution of (6) and (11) is known, it is impossible to compute the exact value of the solution at a fixed time, because of the randomness of Brownian motion. The author of [33] has used a Matlab program to simulate the exact solution of one-dimensional linear stochastic differential equations. The codes in the program produce a discretized Brownian path $W_{i}(i=$ $1,2, \ldots, N)$ with the help of the computer. The value of Brownian motion $B(t)$ at $t=t_{k}$ is obtained by $B\left(t_{k}\right)=$ $\sum_{l=1}^{k-1} W_{l}$. Then it is possible to derive the value of exact solution $x(t)$ at $t=t_{k}$. Such sum must be calculated in every step of the iteration, increasing the amount of computation needed. However, the scheme proposed in this paper can overcome such a problem.

\section{An Application to the Kubo Oscillator}

Consider the Kubo oscillator [34]:

$$
\begin{array}{ll}
d p(t)=-a q(t) d t-b q(t) \circ d B(t), & p(0)=p_{0} \in R, \\
d q(t)=a p(t) d t+b p(t) \circ d B(t), & q(0)=q_{0} \in R,
\end{array}
$$

where $a$ and $b$ are constants and $B(t)$ is a standard one-dimensional Brownian motion. The small circle "o" before $d B(t)$ denotes stochastic differential equations of Stratonovich type. Using (14), the exact solution to (16) is illustrated as follows.

Theorem 4. The exact solution of (16) is given by

$$
\begin{aligned}
& p(t)=p_{0} \cos (a t+b B(t))-q_{0} \sin (a t+b B(t)), \\
& q(t)=p_{0} \sin (a t+b B(t))+q_{0} \cos (a t+b B(t)) .
\end{aligned}
$$

Further, the exact finite-difference scheme for (16) can be constructed by applying (17). Assume $h$ is the time increment 
and $\Delta B_{n}=B\left(t_{n+1}\right)-B\left(t_{n}\right)$ is independent $N(0, h)$-distributed Gaussian random variables. $P_{n}$ and $Q_{n}(n=0,1,2, \ldots)$ are the exact discrete values to $p(t)$ and $q(t)$ when $t=t_{n}=n h$. The exact finite-difference scheme of (16) is given by

$$
\begin{aligned}
& P_{n+1}=p\left(t_{n+1}\right) \\
& =p_{0} \cos \left(a t_{n+1}+b B\left(t_{n+1}\right)\right) \\
& -q_{0} \sin \left(a t_{n+1}+b B\left(t_{n+1}\right)\right) \\
& =p_{0} \cos \left(a t_{n}+b B\left(t_{n}\right)+a h+b \Delta B_{n}\right) \\
& -q_{0} \sin \left(a t_{n}+b B\left(t_{n}\right)+a h+b \Delta B_{n}\right) \\
& =p_{0} \cos a_{n} \cos b_{n}-p_{0} \sin a_{n} \sin b_{n} \\
& -q_{0} \sin a_{n} \cos b_{n}-q_{0} \cos a_{n} \sin b_{n} \\
& =\left(p_{0} \cos a_{n}-q_{0} \sin a_{n}\right) \cos b_{n} \\
& -\left(p_{0} \sin a_{n}+q_{0} \cos a_{n}\right) \sin b_{n} \\
& =P_{n} \cos b_{n}-Q_{n} \sin b_{n} \text {, } \\
& Q_{n+1}=q\left(t_{n+1}\right) \\
& =p_{0} \sin \left(a t_{n+1}+b B\left(t_{n+1}\right)\right) \\
& +q_{0} \cos \left(a t_{n+1}+b B\left(t_{n+1}\right)\right) \\
& =p_{0} \sin \left(a t_{n}+b B\left(t_{n}\right)+a h+b \Delta B_{n}\right) \\
& +q_{0} \cos \left(a t_{n}+b B\left(t_{n}\right)+a h+b \Delta B_{n}\right) \\
& =p_{0} \sin a_{n} \cos b_{n}+p_{0} \cos a_{n} \sin b_{n} \\
& +q_{0} \cos a_{n} \cos b_{n}-q_{0} \sin a_{n} \sin b_{n} \\
& =\left(p_{0} \sin a_{n}+q_{0} \cos a_{n}\right) \cos b_{n} \\
& +\left(p_{0} \cos a_{n}-q_{0} \sin a_{n}\right) \sin b_{n} \\
& =Q_{n} \cos b_{n}+P_{n} \sin b_{n} \text {, }
\end{aligned}
$$

where $a_{n}=a t_{n}+b B\left(t_{n}\right)$ and $b_{n}=a h+b \Delta B_{n}$.

Due to

$$
\begin{aligned}
& \frac{d \gamma(p(t), q(t))}{d t} \\
& =\frac{d\left(p(t)^{2}+q(t)^{2}\right)}{d t} \\
& =2 p(t) \dot{p}(t)+2 q(t) \dot{q}(t) \\
& =2 p(t)(-a q(t)-b q(t) \circ \dot{B}(t)) \\
& \quad+2 q(t)(a p(t)+b p(t) \circ \dot{B}(t)) \\
& =0,
\end{aligned}
$$

then $\gamma(p, q)=p^{2}+q^{2}$ is conservative along the phase flow of (16). That is, $\gamma(p, q)$ is a first integral of $(16)$, indicating that a phase trajectory of $(16)$ is a circle with the center at $(0,0)$ and with the radius $\sqrt{H\left(p_{0}, q_{0}\right)}$. It can be proved that scheme (18) can preserve the first integral $\gamma(p, q)$ exactly.

Theorem 5. Scheme (18) for solving (16) has the property $\gamma\left(P_{n+1}, Q_{n+1}\right)=\gamma\left(P_{n}, Q_{n}\right)$ for any $n=0,1,2, \ldots$

Proof. Substitution of (18) into $\gamma(p, q)$ yields

$$
\begin{aligned}
\gamma\left(P_{n+1}, Q_{n+1}\right)= & P_{n+1}^{2}+Q_{n+1}^{2} \\
= & \left(P_{n} \cos b_{n}-Q_{n} \sin b_{n}\right)^{2} \\
& +\left(Q_{n} \cos b_{n}+P_{n} \sin b_{n}\right)^{2} \\
= & P_{n}^{2} \cos ^{2} b_{n}+Q_{n}^{2} \sin ^{2} b_{n} \\
& +Q_{n}^{2} \cos ^{2} b_{n}+P_{n}^{2} \sin ^{2} b_{n} \\
= & P_{n}^{2}+Q_{n}^{2} .
\end{aligned}
$$

This completes the proof.

It is obvious that (16) is a stochastic Hamiltonian system with $H(p, q)=a\left(p^{2}+q^{2}\right) / 2$ and $H_{1}(p, q)=b\left(p^{2}+q^{2}\right) / 2$, and thus the phase flow of (16) preserves the symplectic structure $d p(t) \wedge d q(t)=d p_{0} \wedge d q_{0}$ for all $t \geq 0$. A good analytical and numerical study of stochastic Hamiltonian systems can be found in $[34,35]$. The following theorem elicits whether scheme (18) is symplectic.

Theorem 6. Scheme (18) for solving (16) preserves the symplectic structure; that is, $d P_{n+1} \wedge d Q_{n+1}=d P_{n} \wedge d Q_{n}$.

Proof. Differentiating (18), it is known that

$$
\begin{aligned}
& d P_{n+1}=\cos b_{n} d P_{n}-\sin b_{n} d Q_{n}, \\
& d Q_{n+1}=\cos b_{n} d Q_{n}+\sin b_{n} d P_{n} .
\end{aligned}
$$

From the exterior product, it can be derived as

$$
\begin{aligned}
d P_{n+1} & \wedge d Q_{n+1} \\
& =\cos ^{2} b_{n} d P_{n} \wedge d Q_{n}-\sin ^{2} b_{n} d Q_{n} \wedge d P_{n} \\
& =d P_{n} \wedge d Q_{n} .
\end{aligned}
$$

This completes the proof.

\section{Numerical Experiments}

A linear stochastic differential equation of Itô type can be written as

$$
\begin{gathered}
d x(t)=2 x(t) d t+x(t) d B(t), \quad t \in[0,1], \\
x(0)=1 .
\end{gathered}
$$

The exact solution to (23) is given by

$$
x(t)=\exp (1.5 t+B(t))
$$




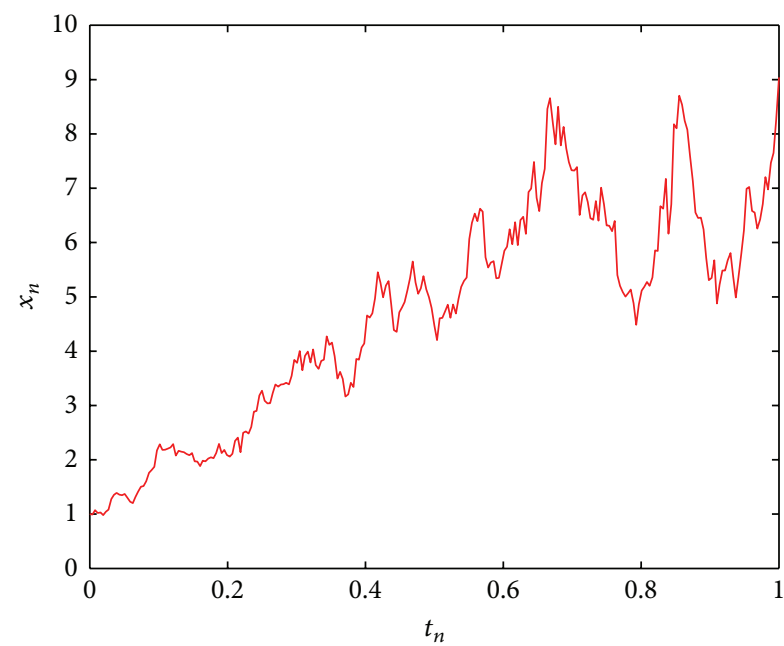

FIGURE 1: The exact solution of (23) simulated by (25) with fixed step size $h=2^{-8}$.

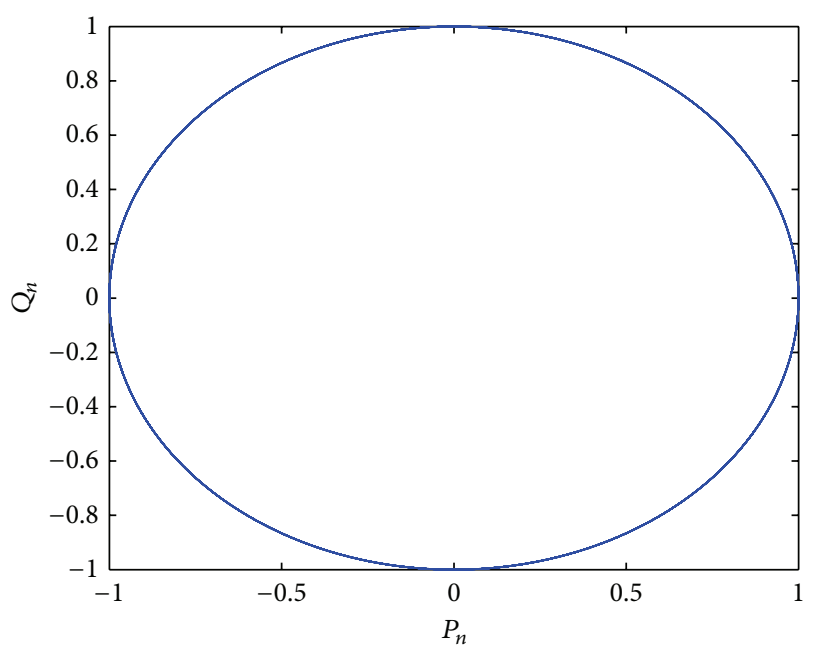

FIGURE 2: Applying (18) to simulate (16) with fixed step size $h=0.02$.

By (8), the exact finite-difference scheme of (23) is

$$
x_{n+1}=\exp \left(1.5 h+\Delta B_{n}\right) x_{n} .
$$

Figure 1 exhibits the exact solution of a sample phase trajectory of (23) simulated by (25).

Next, the exact finite-difference scheme (18) can be applied to solve the Kubo oscillator (16). The coefficients of (16) are chosen as $a=0.2, b=0.01, p_{0}=1, q_{0}=$ $0, h=0.02$, and $t \in[0,400]$. Figure 2 exhibits the numerical solutions of a sample phase trajectory of (16) simulated by (18). Figure 3 shows that the numerical solutions created by (18) could preserve the first integral $\gamma(p, q)$ of (16).

\section{Conclusions}

In this paper, the authors extend the exact finite-difference schemes to linear stochastic differential equations with constant coefficients. The exact finite-difference schemes

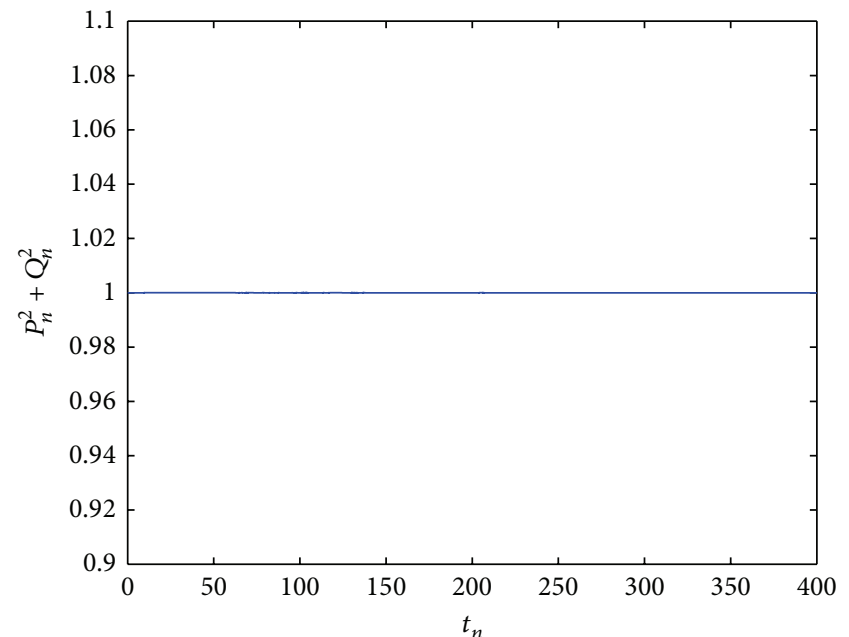

Figure 3: Preservation of first integral $\gamma(p, q)$ by numerical solutions produced by (18).

have been calculated for general $d$-dimensional Itô and Stratonovich type stochastic differential equations. By using the exact finite-difference schemes to solve the Kubo oscillator, the authors have proven that the findings illustrated in this paper can preserve the symplectic structure and first integral. Numerical examples demonstrate the validity of the exact finite-difference schemes in this paper.

\section{Acknowledgment}

This work is supported by the National Science Council of the Republic of China, under Project No. NSC 71031003.

\section{References}

[1] L. P. Blenman, R. S. Cantrell, R. E. Fennell et al., "An alternative approach to stochastic calculus for economic and financial models," Journal of Economic Dynamics \& Control, vol. 19, no. 3, pp. 553-568, 1995.

[2] F. G. Ball and O. D. Lyne, "Optimal vaccination policies for stochastic epidemics among a population of households," Mathematical Biosciences, vol. 177-178, pp. 333-354, 2002.

[3] M. Liu and K. Wang, "Global stability of a nonlinear stochastic predator-prey system with Beddington-DeAngelis functional response," Communications in Nonlinear Science and Numerical Simulation, vol. 16, no. 3, pp. 1114-1121, 2011.

[4] X. Mao, Stochastic Differential Equations and Their Applications, Horwood Publishing Series in Mathematics \& Applications, Horwood Publishing, Chichester, UK, 1997.

[5] B. Øksendal, Stochastic Differential Equations, Springer, Berlin, Germany, 2005.

[6] P. E. Kloeden and E. Platen, Numerical Solution of Stochastic Differential Equations, vol. 23 of Applications of Mathematics, Springer, Berlin, Germany, 1992.

[7] A. Rathinasamy, B. Yin, and B. Yasodha, "Numerical analysis for stochastic age-dependent population equations with Poisson jump and phase semi-Markovian switching," Communications in Nonlinear Science and Numerical Simulation, vol. 16, no. 1, pp. 350-362, 2011. 
[8] P. Wang, "Three-stage stochastic Runge-Kutta methods for stochastic differential equations," Journal of Computational and Applied Mathematics, vol. 222, no. 2, pp. 324-332, 2008.

[9] D. J. Higham, X. Mao, and A. M. Stuart, "Strong convergence of Euler-type methods for nonlinear stochastic differential equations," SIAM Journal on Numerical Analysis, vol. 40, no. 3, pp. 1041-1063, 2002.

[10] I. Shoji, "A note on convergence rate of a linearization method for the discretization of stochastic differential equations," Communications in Nonlinear Science and Numerical Simulation, vol. 16, no. 7, pp. 2667-2671, 2011.

[11] L. Hu and S. Gan, "Convergence and stability of the balanced methods for stochastic differential equations with jumps," International Journal of Computer Mathematics, vol. 88, no. 10, pp. 2089-2108, 2011.

[12] X. Wang and S. Gan, "Compensated stochastic theta methods for stochastic differential equations with jumps," Applied Numerical Mathematics, vol. 60, no. 9, pp. 877-887, 2010.

[13] F. Jiang, Y. Shen, and L. Liu, "Taylor approximation of the solutions of stochastic differential delay equations with Poisson jump," Communications in Nonlinear Science and Numerical Simulation, vol. 16, no. 2, pp. 798-804, 2011.

[14] F. Jiang, Y. Shen, and F. Wu, "A note on order of convergence of numerical method for neutral stochastic functional differential equations," Communications in Nonlinear Science and Numerical Simulation, vol. 17, no. 3, pp. 1194-1200, 2012.

[15] G. N. Milstein, Numerical Integration of Stochastic Differential Equations, vol. 313 of Mathematics and Its Applications, Kluwer Academic Publishers, Dordrecht, The Netherlands, 1995.

[16] R. E. Mickens, Nonstandard Finite Difference Models of Differential Equations, World Scientific Publishing, River Edge, NJ, USA, 1994.

[17] T. E. Simos and J. Vigo-Aguiar, "A dissipative exponentiallyfitted method for the numerical solution of the Schrödinger equation and related problems," Computer Physics Communications, vol. 152, no. 3, pp. 274-294, 2003.

[18] T. E. Simos and J. Vigo-Aguiar, "An exponentially-fitted high order method for long-term integration of periodic initial-value problems," Computer Physics Communications, vol. 140, no. 3, pp. 358-365, 2001.

[19] A. Tocino and J. Vigo-Aguiar, "Symplectic conditions for exponential fitting Runge-Kutta-Nyström methods," Mathematical and Computer Modelling, vol. 42, no. 7-8, pp. 873-876, 2005.

[20] R. B. Potts, "Differential and difference equations," The American Mathematical Monthly, vol. 89, no. 6, pp. 402-407, 1982.

[21] R. P. Agarwal, Difference Equations and Inequalities: Theory, Methods, and Applications, vol. 228 of Monographs and Textbooks in Pure and Applied Mathematics, Marcel Dekker, New York, NY, USA, 2nd edition, 2000.

[22] J. Vigo-Aguiar and J. M. Ferrándiz, "A general procedure for the adaptation of multistep algorithms to the integration of oscillatory problems," SIAM Journal on Numerical Analysis, vol. 35, no. 4, pp. 1684-1708, 1998.

[23] J. L. Cieśliński, "Comment on 'conservative discretizations of the Kepler motion," Journal of Physics A, vol. 43, no. 22, Article ID 228001, 4 pages, 2010.

[24] J. L. Cieśliński, "On the exact discretization of the classical harmonic oscillator equation," Journal of Difference Equations and Applications, vol. 17, no. 11, pp. 1673-1694, 2011.

[25] T. Sakamoto, N. Hori, and Y. Ochi, "Exact linearization and discretization of nonlinear systems satisfying a lagrange pde condition," Transactions of the Canadian Society for Mechanical Engineering, vol. 35, no. 2, pp. 215-228, 2011.

[26] R. E. Mickens and T. M. Washington, "A note on exact finite difference schemes for the differential equations satisfied by the Jacobi cosine and sine functions," Journal of Difference Equations and Applications, vol. 19, no. 6, pp. 1042-1047, 2013.

[27] L.-I. W. Roeger and R. E. Mickens, "Exact finite difference and non-standard finite difference schemes for $d x / d t=-\lambda x^{\alpha}$," Journal of Difference Equations and Applications, vol. 18, no. 9, pp. 1511-1517, 2012.

[28] L.-I. W. Roeger and R. E. Mickens, "Exact finite-difference schemes for first order differential equations having three distinct fixed-points," Journal of Difference Equations and Applications, vol. 13, no. 12, pp. 1179-1185, 2007.

[29] L.-I. W. Roeger, "Exact finite-difference schemes for twodimensional linear systems with constant coefficients," Journal of Computational and Applied Mathematics, vol. 219, no. 1, pp. 102-109, 2008.

[30] T. Simos, "The exact discrete model of a third-order system of linear stochastic differential equations with observable stochastic trends," Macroeconomic Dynamics, vol. 13, no. 5, pp. 656-672, 2009.

[31] H. Schurz, "Numerical analysis of stochastic differential equations without tears," in Handbook of Stochastic Analysis and Applications, V. Lakshmikantham and D. Kannan, Eds., pp. 237359, Dekker, New York, NY, USA, 2002.

[32] R. García-Rubio, "Accurate numerical solution of Black-Scholes option pricing equations," International Journal of Financial Markets and Derivatives, vol. 2, no. 3, pp. 236-243, 2011.

[33] D. J. Higham, "An algorithmic introduction to numerical simulation of stochastic differential equations," SIAM Review, vol. 43, no. 3, pp. 525-546, 2001.

[34] G. N. Milstein, Yu. M. Repin, and M. V. Tretyakov, "Numerical methods for stochastic systems preserving symplectic structure," SIAM Journal on Numerical Analysis, vol. 40, no. 4, pp. 1583-1604, 2002.

[35] G. N. Milstein, Yu. M. Repin, and M. V. Tretyakov, "Symplectic integration of Hamiltonian systems with additive noise," SIAM Journal on Numerical Analysis, vol. 39, no. 6, pp. 2066-2088, 2002. 


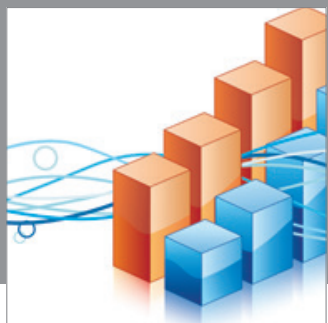

Advances in

Operations Research

mansans

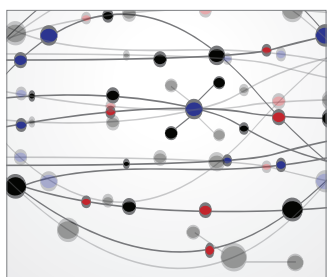

The Scientific World Journal
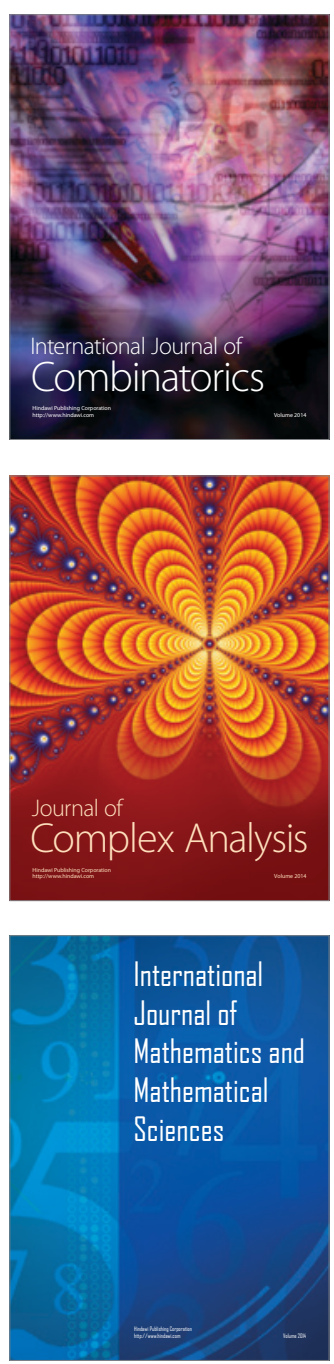
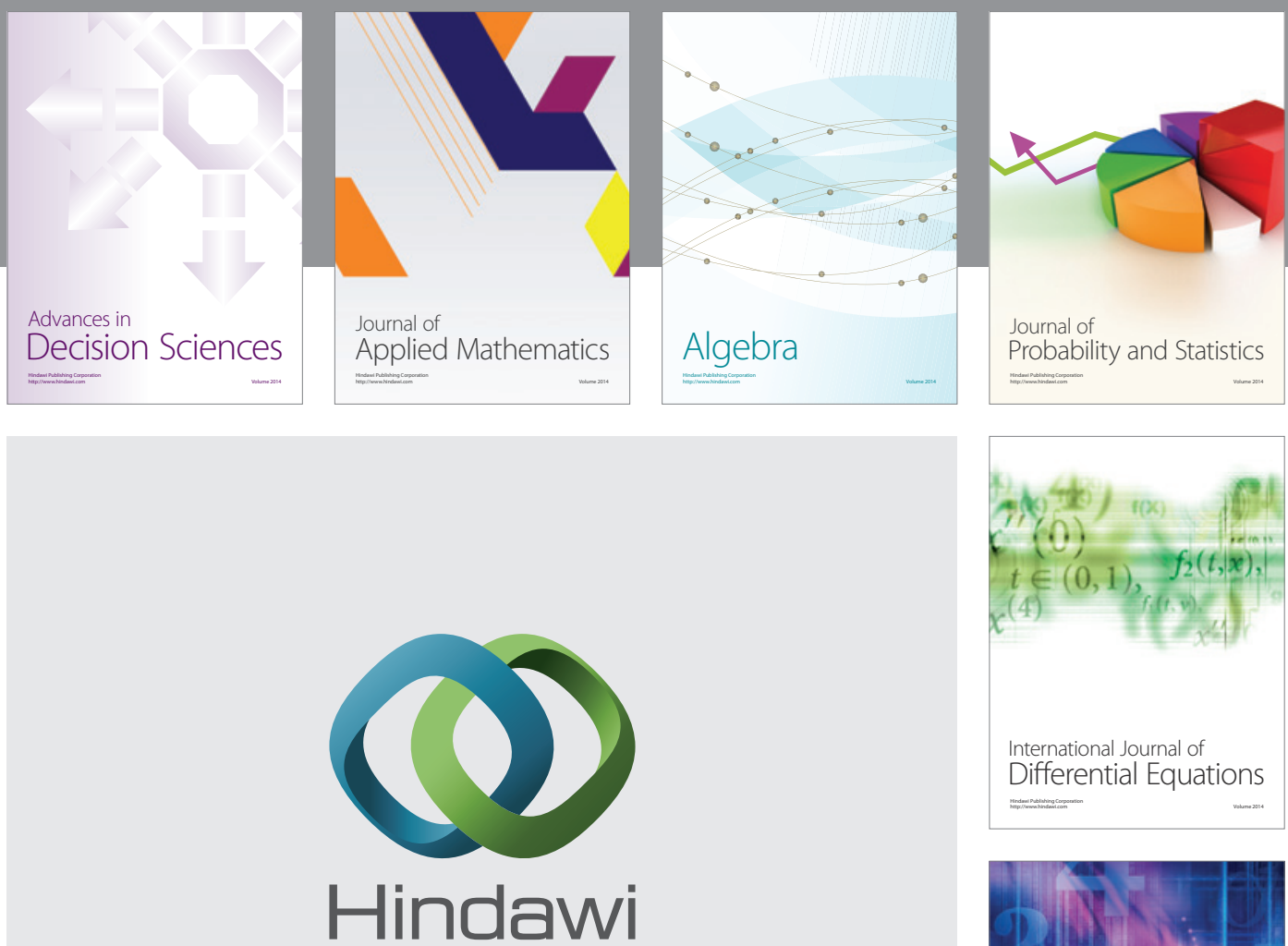

Submit your manuscripts at http://www.hindawi.com
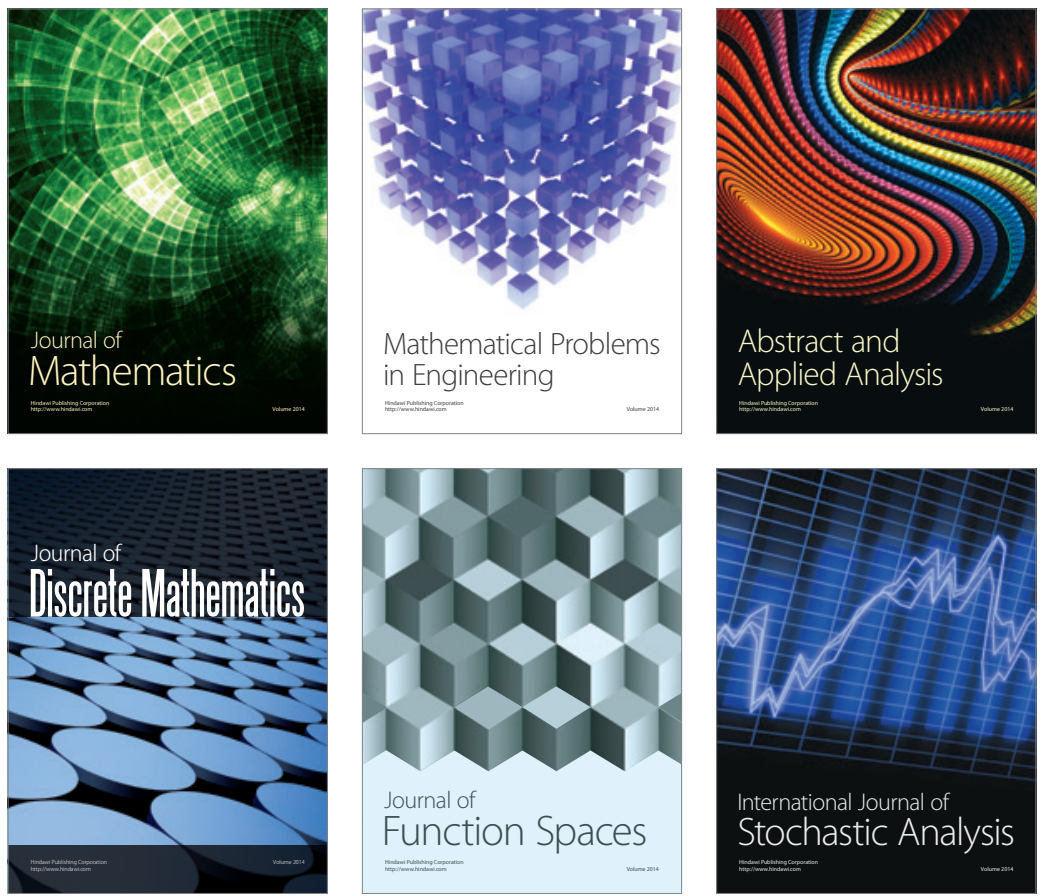

Journal of

Function Spaces

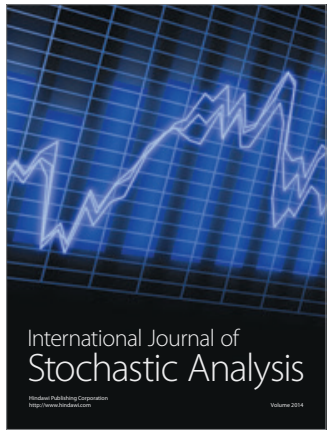

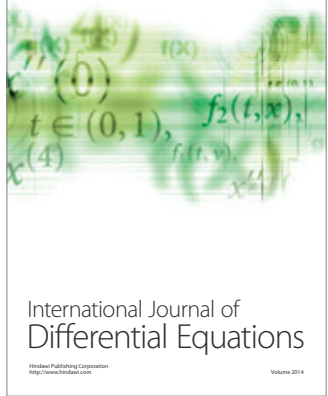
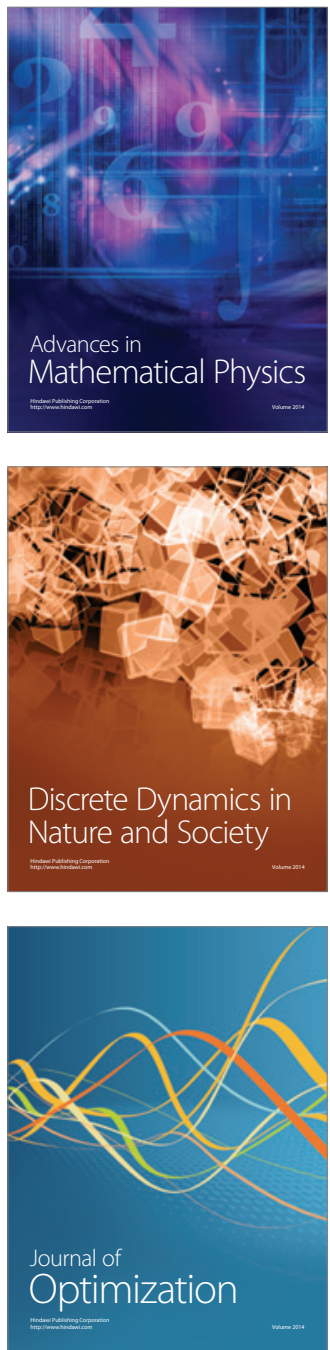